\title{
Desenvolvimento de metodologia para avaliação da adesividade agregado-ligante com o uso de processamento digital de imagem
}

\author{
Jorge Luiz Oliveira Lucas Júnior ${ }^{1}$, Jorge Barbosa Soares ${ }^{2}$
}

${ }^{1}$ Universidade Federal do Ceará \& Faculdade Ari de Sá, Fortaleza, CE, Brasil, j.lucas.j@det.ufc.br 2Universidade Federal do Ceará, Fortaleza, CE, Brasil, jsoares@det.ufc.br

\section{Recebido:}

13 de dezembro de 2017

Aceito para publicação:

2 de abril de 2018

Publicado:

30 de abril de 2019

Editor de área:

Kamilla Vasconcelos

\section{Palavras-chaves:}

Adesividade,

Processamento Digital de Imagens,

Dano por Umidade.

\section{Keywords:}

Adhesiveness,

Digital Image Processing,

Moisture Damage.

DOI:10.14295/transportes.v27i1.1552

\begin{abstract}
RESUMO
A adesividade na interface agregado-ligante de misturas asfálticas é uma propriedade importante de se avaliar de modo a prevenir danos relacionados a umidade em pavimentos asfálticos. No Brasil, um dos ensaios normatizados para analisar essa propriedade é denominado Agregado graúdo: Determinação da adesividade ao ligante betuminoso (NBR 12583-2017), que proporciona um resultado qualitativo (satisfatório ou insatisfatório). A presente pesquisa visa desenvolver uma metodologia usando processamento digital de imagens a partir de imagens obtidas de smartphone que possibilite a determinação do percentual de área de agregado recoberto pelo ligante asfáltico, tornando o resultado do ensaio quantitativo. Foram analisadas 37 imagens para a calibração, além da análise de 5 imagens padrão, cujas dimensões são conhecidas, para comparação com as áreas obtidas por meio da presente metodologia. Os resultados indicaram ser possível quantificar o percentual de área do agregado recoberto pelo ligante, além da identificação dos agregados outliers. A metodologia mostrou-se promissora e executável em curto prazo, tendo em vista a rapidez de execução e a precisão dos resultados, podendo auxiliar na caracterização e seleção adequada de materiais para uso em pavimentação asfáltica.
\end{abstract}

\section{ABSTRACT}

Adhesiveness at the aggregate-binder interface of asphalt mixtures is an important property to be evaluated in order to prevent moisture damage in asphalt pavements. In Brazil, one of the standards to analyze this property is the test Coarse Aggregate: Determination of bituminous coating (NBR 12583-2017), which provides qualitative results (satisfactory or unsatisfactory). This research aims to develop a methodology using digital images processing from smartphone that allows the determination of the percentage area of the aggregate covered by the asphaltic binder. Thirty-seven images were analyzed for the calibration, and five common images of known dimensions were used for comparison with the areas obtained in the present methodology. The results indicated that it is possible to quantify the aggregate area covered by the binder along with the identification of outliers. It is shown the potential of the methodology which can be implemented in the short term, given the duration and accuracy of the procedure. This may help in the characterization and adequate selection of materials for use in the asphalt paving industry.

\section{INTRODUÇÃO}

Em uma mistura asfáltica usada em revestimentos, a adesividade é caracterizada pela resistência adesiva e coesiva da mistura contra o deslocamento de agregado, fixados pelo ligante asfáltico (Abeda, 2010). 
A adesividade agregado-ligante é afetada diretamente pela umidade (Lottman, 1978). Os danos causados pela umidade não estão relacionados somente com a quebra da ligação agregadoligante, mas também atuam como um catalisador de defeitos, como a deformação permanente e o trincamento por fadiga (Cho e Kim, 2010; Hamedia e Tahamib, 2018). Apesar da importância desse tema na área da pavimentação, os protocolos de análise dessa propriedade ainda são limitados.

A NBR 12583-2017 indica que se a película betuminosa tiver recoberto totalmente os agregados, o resultado é satisfatório. Por outro lado, se o recobrimento for parcial, a adesividade é considerada insatisfatória. Segundo essa norma, não há a preocupação em quantificar a área de agregado recoberta pelo ligante para considerar a adesividade satisfatória ou ainda ranquear agregados minerais e ligantes asfálticos distintos quanto à compatibilidade para produzir uma adequada adesividade.

Diante desse contexto, o objetivo desta pesquisa é desenvolver uma metodologia baseada no Processamento Digital de Imagens (PDI), que possibilite a determinação do percentual de área de agregado graúdo, compreendido entre as peneiras de $19 \mathrm{~mm}$ e 12,5 mm, recoberta pelo ligante asfáltico, buscando uma resposta quantitativa para o ensaio de adesividade.

\section{REVISÃO DE LITERATURA}

\subsection{Adesividade Agregado-Ligante}

Curtis et al. (1993) mencionam que a sensibilidade à água é um fator chave na adesão do asfalto com o agregado. Huang et al. (2016) afirmam que o efeito da água no pavimento exerce forte influência na interface agregado-ligante.

Para investigar a susceptibilidade à umidade de misturas asfálticas há vários ensaios laboratoriais reportados na literatura (Liang, 2008). Apesar da maioria desses não simular inteiramente as condições de campo, eles podem indicar se há deslocamento de asfalto da superfície do agregado quando em contato com a água.

Um dos ensaios normatizados no Brasil para análise da adesividade é a NBR 12583-2017. A adesividade é definida como a propriedade do agregado ser aderido por material betuminoso. A análise é verificada pelo não deslocamento da película betuminosa que recobre o agregado. Assim, quando o resultado do ensaio de adesividade não é satisfatório, como visto na Figura 1 (a), é necessária a utilização de aditivos melhoradores de adesividade (AMO) para aumentar a compatibilidade entre os agregados e o ligante asfáltico, tornando a adesividade satisfatória [Figura $1(b)]$.

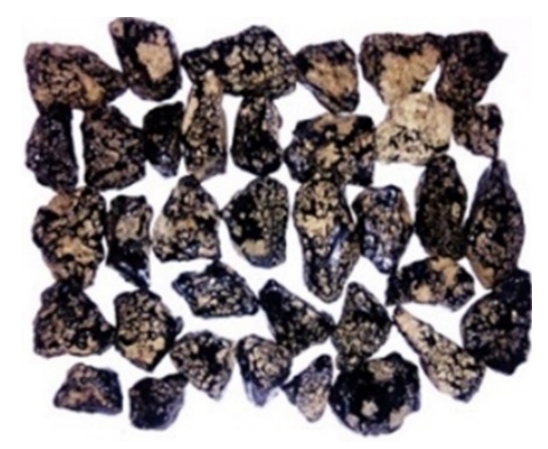

(a)

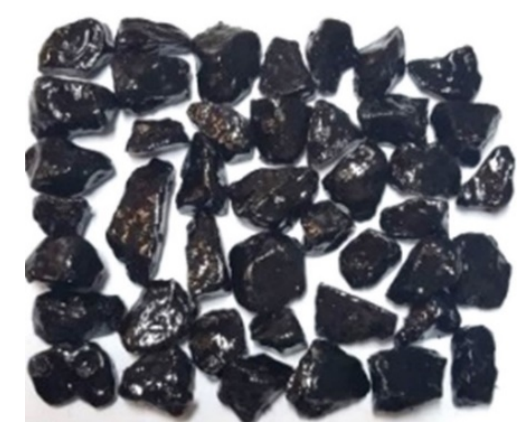

(b)

Figura 1. Resultado do ensaio de adesividade ao agregado graúdo. (a) Agregado com CAP puro (b) Agregado com AMO 


\subsection{Processamento Digital de Imagens}

Uma imagem pode ser definida como uma função bidimensional, $f(x, y)$, em que $x$ e $y$ são coordenadas espaciais (plano), e a amplitude de $f$ em qualquer par de coordenadas $(x, y)$ é chamada de intensidade ou nível de cinza da imagem nesse ponto. Quando $x, y$ e os valores de intensidade de $f$ são quantidades finitas e discretas, denomina-se de imagem digital (Gonzales e Woods, 2010).

De acordo com Marques Filho e Vieira Neto (1999), uma imagem digital pode ser vista como uma matriz, conforme a Equação (1), cujas linhas e colunas identificam um ponto na imagem e o valor corresponde ao nível de cinza da imagem naquele ponto.

$$
f(x, y)=\left[\begin{array}{cccc}
f(1,1) & f(1,2) & \cdots & f(1, m) \\
f(2,1) & f(2,2) & \cdots & f(2, m) \\
\vdots & \vdots & \vdots & \vdots \\
f(n, 1) & f(n, 2) & \cdots & f(n, m)
\end{array}\right]
$$

Para transformar uma imagem contínua [Figura 2 (a)], em uma imagem digital [Figura 2 (b)], é necessário realizar a discretização espacial (amostragem) e a discretização em amplitude (quantização) (Aura Conci e Leta, 2008). Conforme Gonzales e Woods (2010), a amostragem converte a imagem analógica em uma matriz de $m$ por $n$ pontos, denominados pixels e a quantização faz com que cada um desses pixels assuma um valor inteiro.

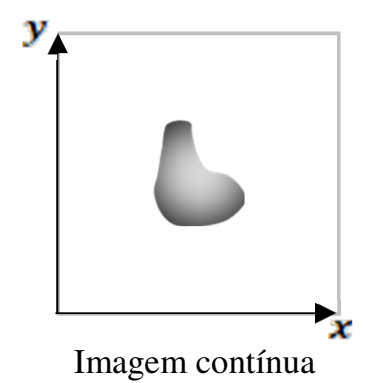

(a)

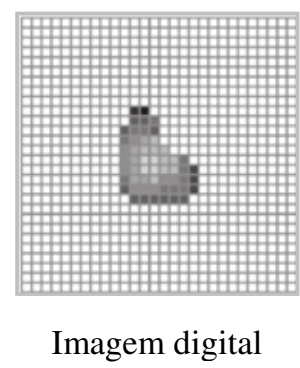

(b)

Figura 2. Transformação da imagem contínua em imagem digital (Adaptada de Gonzales e Woods, 2010).

Segundo Silva (2001), a função primordial do PDI é facilitar a identificação e a extração da informação contida nas imagens, para posterior interpretação. Albuquerque et al. (2012) indicam que o PDI tem diversas etapas, como: formação e aquisição da imagem, digitalização, préprocessamento, segmentação, pós-processamento, extração de atributos, classificação e reconhecimento.

A segmentação tem por objetivo particionar a imagem de modo que seja possível explicitar suas regiões de interesse, agrupando seus pixels de forma que eles se destaquem dos demais (Peres, 2010). A segmentação consiste em separar as regiões de uma imagem quando esta apresenta duas classes: o fundo e o objeto. As estratégias de segmentação são baseadas em similaridade ou descontinuidade entre pixels (Cavalcante, 2010 e Zaitouna e Aqelb, 2015).

Uma das principais técnicas de segmentação baseada em similaridade é a limiarização. Pelo fato da limiarização produzir uma imagem binária à saída, o processo também é denominado, binarização. A forma mais simples de limiarização consiste na bipartição do histograma, convertendo os pixels cujo tom de cinza é maior ou igual a certo valor de limiar (T) em brancos e os demais em pretos, conforme Figura 3 (Marques Filho e Vieira Neto, 1999). 


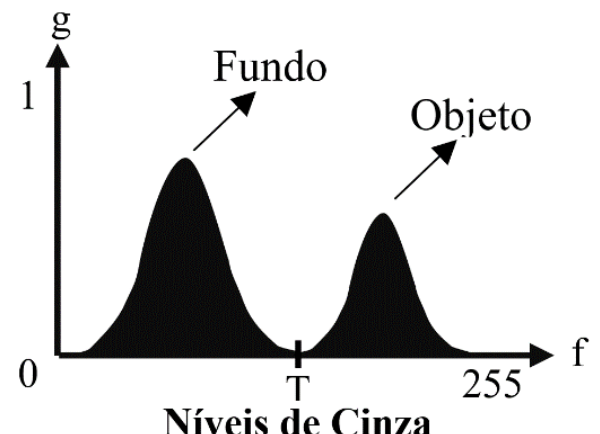

Figura 3. Bipartição do histograma (Marques Filho e Vieira Neto, 1999).

Segundo Sonka (1998), matematicamente, a limiarização pode ser descrita como uma técnica de processamento de imagens na qual uma imagem de entrada $f(x, y)$ de $N$ níveis de cinza produz à saída uma imagem $g(x, y)$, chamada de imagem limiarizada, cujo número de níveis de cinza é menor que $N$. Normalmente, $g(x, y)$ apresenta dois níveis de cinza, em que os pixels rotulados com 1 correspondem aos objetos e os pixels rotulados com 0 correspondem ao fundo (background); Tal descrição está representada na Equação 2:

$$
\begin{aligned}
g(x, y) & =1 \text { se } f(x, y) \geq T \\
& =0 \text { se } f(x, y)<T
\end{aligned}
$$

em que $\quad T: \quad$ valor de tom de cinza, denominado limiar;

\subsection{PDI em Misturas Asfálticas}

O PDI tem se tornado uma importante ferramenta para análise das propriedades dos materiais que compõem a mistura asfáltica. Vasconcelos et al. (2005) analisaram a estrutura interna de misturas asfálticas por meio do software Imagetool. Concluiu-se que quanto menor o tamanho dos agregados, mais uniforme tende a ser a distribuição destes.

Bessa et al. (2011) mediram o polimento, a abrasão e a quebra dos agregados usando o Aggregate Imaging Measurement System (AIMS). Zelelew e Papagiannakis (2011) implementaram um algoritmo de limiarização para PDI de misturas asfálticas, usando o software MATLAB.

Gouveia et al. (2015) utilizaram a esqueletização multiescala para determinar as características de forma dos agregados. Conclui-se que esta técnica é promissora, pois as duas classes de agregados analisadas, basalto e seixo, formaram esqueletos bem diferenciados.

\section{MATERIAIS E MÉTODOS}

O fluxograma que representa a metodologia e o respectivo código da programação estão dispostos nas Figuras A1 e A2 no Anexo.

\subsection{Aquisição de imagens}

Os agregados recobertos pelo ligante (chamados de mistura) após o ensaio de adesividade (NBR 12583-2017) são colocados sobre uma folha de fundo branco em cima de uma superfície plana. A imagem da mistura foi obtida pela câmera de um smartphone de 13 megapixels e resolução de 720 x 1280. 0 celular foi mantido o mais paralelo possível ao plano (manualmente) em que se encontrava a amostra, contudo sem um dispositivo especial que garantisse o paralelismo. As imagens obtidas são salvas em formato Joint Photographic Experts Group (.JPG). 


\subsection{Imagens utilizadas para comparação}

Devido à dificuldade em se estimar a área de recobrimento após o ensaio em questão, decidiuse utilizar 5 imagens com dimensões conhecidas (imagens padrão) para comparar com a metodologia proposta. As partes pretas da imagem simulam o ligante asfáltico, enquanto que as partes marrons simulam o agregado [Figura 4]. Conhecendo-se as dimensões dessas imagens, é possível determinar seus respectivos percentuais teóricos de área recoberta (AтеóRICA). Os procedimentos descritos a seguir foram utilizados para as 5 imagens, sendo a Figura 4 (a) usada como exemplo na explicação.

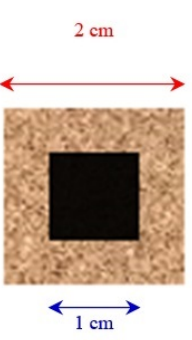

(a)

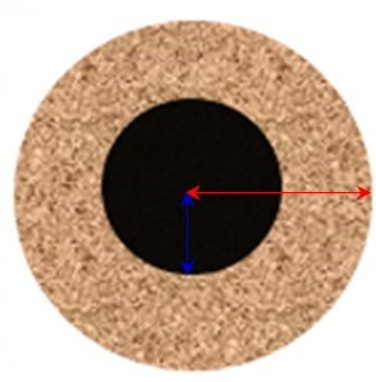

(b)

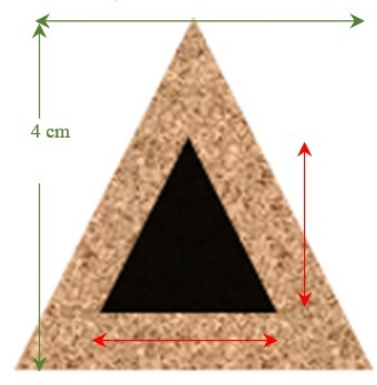

(c)

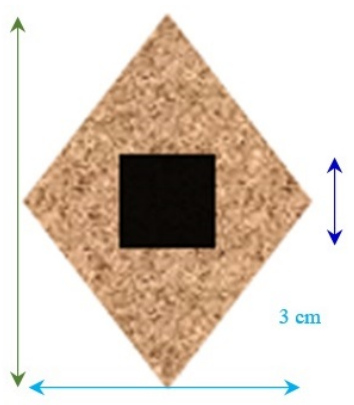

(d)

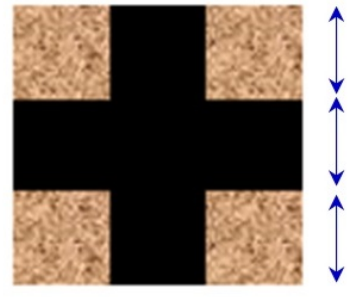

(e)

Figura 4. Imagens padrão utilizadas para comparação.

\subsection{Pré-processamento/Realce}

As etapas seguintes foram executadas no software MATLAB R2017a, conforme as Figuras A1 e A2. Como as imagens utilizadas para validação e a imagem obtida após o ensaio de adesividade têm padrão RGB (Red, Green, Blue) é imprescindível a utilização da função RGB2GRAY contida na Toolbox do MATLAB, transformando-as em imagens monocromáticas [Figura (5)].

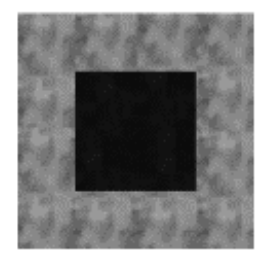

Figura 5. Imagem monocromática.

A distribuição de pixels da Figura 5 foi feita e obteve-se como resultado o histograma da Figura 6. 


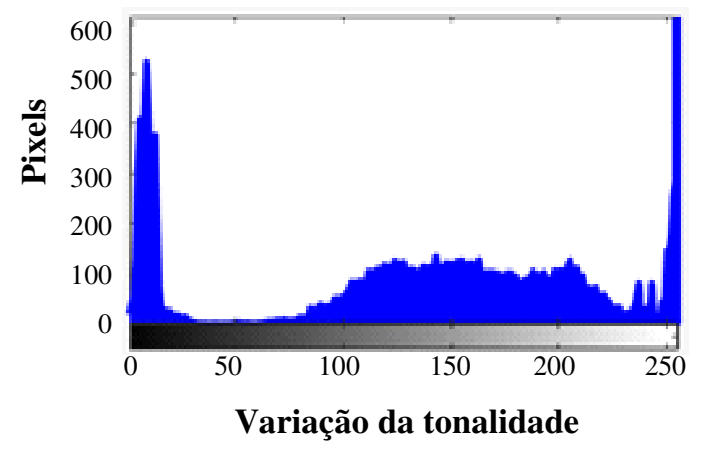

Figura 6. Histograma de distribuição de pixels da imagem.

Por meio do histograma é possível inferir os limiares. Esses limiares (T) são obtidos nos vales do histograma. Na Figura 6, em $0<\mathrm{T} \leq 25$, (na abscissa que indica a variação da tonalidade), se encontram os pixels do ligante asfáltico, representados na Figura 5 pela cor preta. Em $25<\mathrm{T} \leq$ 50 existe uma grande depressão na qual não se encontram pixels. Já em $50<\mathrm{T} \leq 240$ tem-se os pixels que representam o agregado na Figura 5. Próximo a 255 encontram-se os pixels brancos representando a folha de fundo branco. Como o histograma foi aumentado, para poder verificar os picos e vales formados, não foi possível visualizar até o limite de pixels brancos. Todavia, o valor das ordenadas referente a esse tom atingiu 11.600 pixels.

\subsection{Segmentação por Limiarização}

Nesta etapa a imagem foi binarizada, sendo que os pixels abaixo do limiar definido tiveram atribuído o valor zero (0), representando a cor preta; e os pixels acima desse limiar tiveram o valor (1), representando a cor branca. Para separar os pixels referentes ao ligante asfáltico pode ser usada a função GRAYTHRESH, que usa o método de Otsu para escolher o limiar automaticamente, e minimiza a variação intraclasse dos pixels pretos e brancos (Otsu, 1979). Entretanto, muitas vezes esse limiar sofre distorções e o caminho mais representativo é o método manual de tentativas. Utilizou-se o limiar de $\mathrm{T} \leq 0,200$, pois esse foi o valor que mais se adequou à imagem analisada. Para separar os pixels brancos utilizou-se $\mathrm{T} \leq 0,785$. Para o primeiro limiar a resposta do percentual de área recoberta pelo ligante pode ser vista na Figura 7.

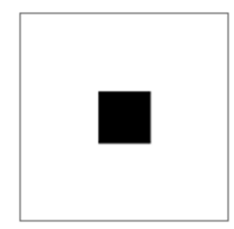

Figura 7. Limiarização da imagem.

No MATLAB, a função utilizada faz o somatório de todos os pixels brancos (0). Todavia, os pixels de interesse são os pretos (1) da Figura 7, então a imagem é invertida conforme visto na Figura 8 (a), permitindo a determinação dos pixels do ligante asfáltico. Para encontrar o número de pixels do agregado é necessário realizar o mesmo procedimento relatado até esse passo, só que dessa vez o limiar utilizado será $\mathrm{T} \leq 0,785$. A imagem gerada corresponderá a área total dos agregados representada na Figura 8 (b) pela área branca. 


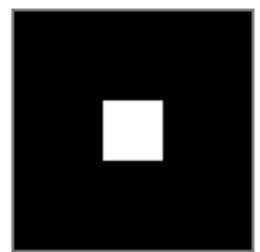

(a)

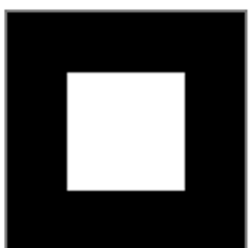

(b)

Figura 8. Inversão dos pixels.

\subsection{Reconhecimento e Classificação}

Com o número de pixels brancos das Figuras 8 (a) e (b) é possível determinar o percentual de área do agregado recoberto pelo ligante $\left(A_{P D I}\right)$, conforme Equação (3).

$$
A_{P D I}=\frac{P_{L}}{P_{A}} \cdot 100
$$

em que $P_{L}: \quad \quad$ é o número de pixels do ligante,

$P_{A}$ : $\quad$ é o número de pixels do agregado.

Os valores obtidos nas análises das imagens da Figura 4 constam na Tabela 1, por meio da qual é possível concluir que o percentual de área determinado pela metodologia proposta é muito próximo ao valor teórico, o que indica que é possível encontrar a área de agregado recoberta pelo ligante após o ensaio de adesividade.

Tabela 1: Comparação da área determinada por PDI com a área teórica

\begin{tabular}{cccccc}
\hline Figura 4 & $\boldsymbol{P}_{\boldsymbol{L}}$ & $\boldsymbol{P}_{\boldsymbol{A}}$ & Limiares & A $_{\text {TEÓRICA }}[\%]$ & A $_{\text {PDI }}[\%]$ \\
\hline (a) Imagem 1 & 1444 & 5754 & & 25,00 & 25,09 \\
(b) Imagem 2 & 4483 & 17919 & & 25,00 & 25,02 \\
(c) Imagem 3 & 2830 & 11284 & $0,200 \mid 0,785$ & 25,00 & 25,08 \\
(d) Imagem 4 & 1444 & 8646 & & 16,66 & 16,70 \\
(e) Imagem 5 & 7212 & 12984 & & 55,55 & 55,55 \\
\hline
\end{tabular}

\subsection{Avaliação da Influência da Escolha de Limiares Manualmente nos Resultados}

A avaliação da influência da escolha dos limiares manuais nos percentuais de área recoberta foi investigada. Para isso, os limiares das 5 imagens foram modificados e os respectivos percentuais de área recoberta foram analisados. Para o limiar 0,200 aplicou-se variações de $\pm 0,025$, ou seja, limiares de 0,175 e de 0,225, abrangendo uma faixa de 0,05. 0 mesmo foi feito para o limiar 0,785 , resultando nos limiares 0,760 e 0,810 . Os resultados referentes a esta análise estão dispostos na Tabela 2.

Tabela 2: Percentuais de área recoberta em função dos limiares

\begin{tabular}{|c|c|c|c|c|c|c|}
\hline & Limiares & (a) Imagem 1 & (b) Imagem 2 & (c) Imagem 3 & (d) Imagem 4 & (e) Imagem 5 \\
\hline \multirow{3}{*}{$2^{2}$} & 0,175 & 1444 & 4470 & 2822 & 1444 & 7171 \\
\hline & 0,200 & 1444 & 4483 & 2830 & 1444 & 7212 \\
\hline & 0,225 & 1444 & 4492 & 2857 & 1444 & 7256 \\
\hline \multirow{6}{*}{2} & 0,760 & 5671 & 17694 & 10998 & 8304 & 12670 \\
\hline & 0,785 & 5754 & 17929 & 11284 & 8646 & 12984 \\
\hline & 0,810 & 5755 & 17919 & 11341 & 8579 & 12755 \\
\hline & Maior APDI [\%] & 25,46 & 25,39 & 25,98 & 17,39 & 56,92 \\
\hline & Menor APDI [\%] & 25,09 & 25,00 & 24,95 & 16,70 & 55,23 \\
\hline & Diferença & 0,37 & 0,39 & 1,03 & 0,69 & 1,61 \\
\hline
\end{tabular}


Analisando as diferentes combinações de $P_{A}$ e de $P_{L}$, conclui-se que mesmo para uma faixa de 0,05 entre os limiares, a maior variação obtida para as 5 imagens foi de 1,61\%, enquanto que a variação média das 5 imagens foi de $0,81 \%$.

\section{RESULTADOS E DISCUSSÕES}

\subsection{PDI Pós-ensaio NBR 12583-2017}

A Figura 9 (a) mostra o resultado do ensaio de adesividade do agregado graúdo. 0 agregado utilizado tem origem granítica e o CAP é classificado como 50/70. Como a imagem é colorida faz-se necessário transformá-la para escala de cinza, conforme Figura 9 (b). Na Figura 9 (c) aplicou-se o limiar de separação do ligante asfáltico. A inversão dos pixels foi realizada, conforme Figura 9 (d), possibilitando a determinação de $P_{L}$. Em seguida, realizou-se o mesmo procedimento com outro limiar, o que permitiu a determinação de $P_{A}$, conforme Figura 9 (e).

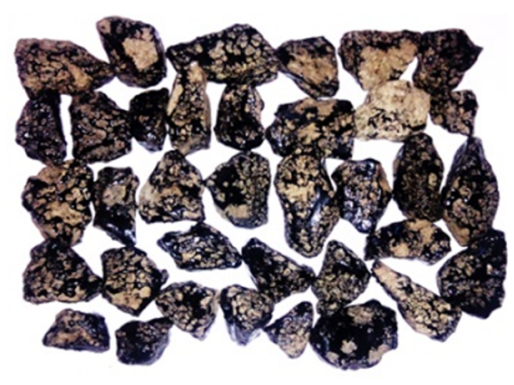

(a)

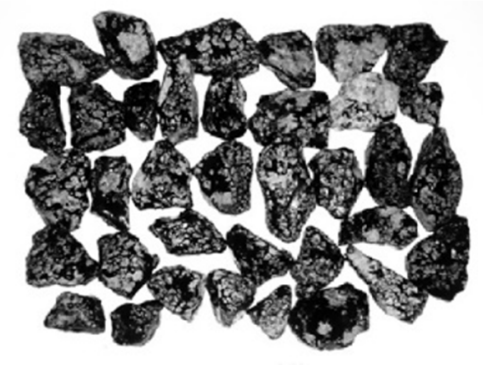

(b)

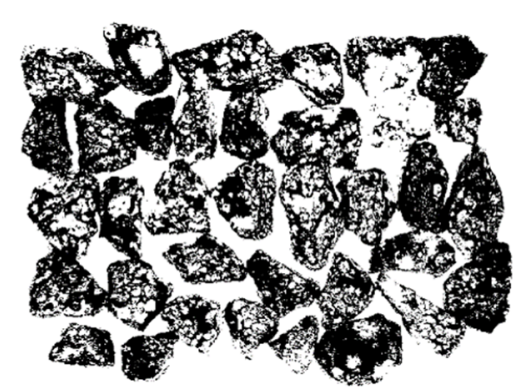

(c)

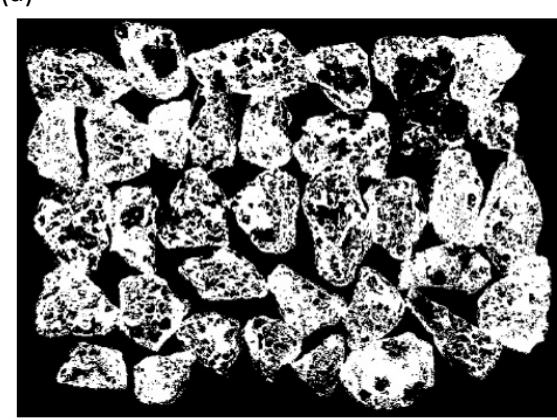

(d)

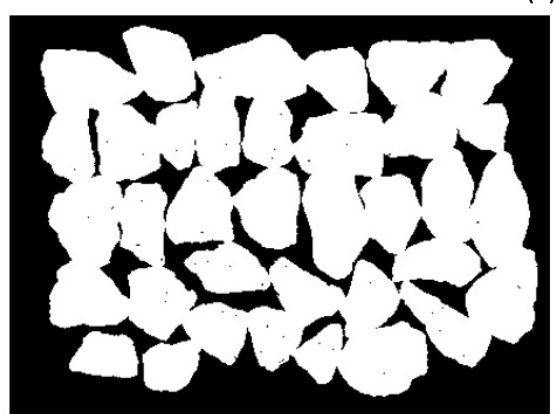

(e)

Figura 9. Processamento e análise das imagens obtidas após o ensaio NBR 12583/2017.

Os agregados recobertos pelo ligante da Figura 9 (a) foram fotografados três vezes. Esses agregados são rotacionados aleatoriamente para minimizar a interferência do operador. Esse procedimento foi realizado pois o ensaio tem um resultado tridimensional e as imagens retiradas são bidimensionais. 0 que se busca com esse procedimento é minimizar o erro de um recobrimento tridimensional para uma imagem bidimensional. Essas três imagens são analisadas e é feita sua média aritmética. Os resultados da análise da Figura 9 (a) e de suas outras duas rotações estão detalhados na Tabela 3

Tabela 3: Áreas de recobrimento do ligante.

\begin{tabular}{|c|c|c|c|c|c|}
\hline & $P_{L}$ & $P_{A}$ & Limiares & $A_{P D I}[\%]$ & $\bar{A}_{P D I}[\%]$ \\
\hline Imagem 1 & 40668 & 80962 & \multirow{3}{*}{$0,200 \mid 0,785$} & 50,23 & \multirow{3}{*}{50,28} \\
\hline Imagem 1" & 40349 & 80452 & & 50,15 & \\
\hline Imagem 1"' & 41126 & 81512 & & 50,45 & \\
\hline
\end{tabular}


Para realizar um teste estatístico, cada agregado foi numerado e separado, e o seu percentual de área de recobrimento foi determinado, conforme Figura 10.

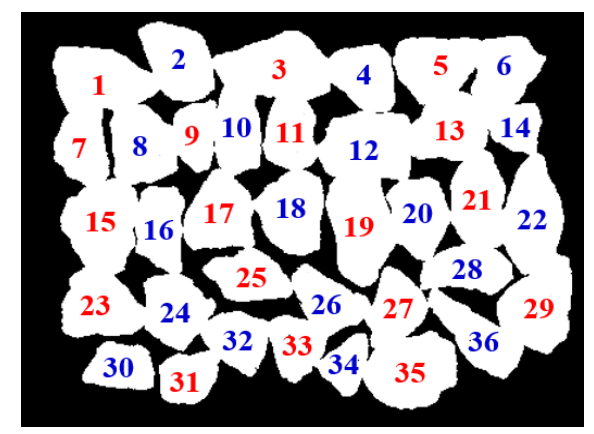

Figura 10. Separação dos agregados.

Analisando as Tabelas 3 e 4 é possível notar que o percentual de área recoberta na imagem com todos os agregados foi de 50,28\%, enquanto que na análise de cada agregado individualmente o somatório foi de 50,42\%. A pequena diferença pode ser atribuída ao contato entre alguns agregados no momento da captação da imagem. Por esse motivo, recomenda-se que os agregados recobertos pelo ligante sejam colocados afastados uns dos outros quando da aquisição das imagens.

Tabela 4: Valores determinados para cada agregado individualmente

\begin{tabular}{|c|c|c|c|c|c|c|c|c|c|}
\hline Agregado & 1 & 2 & 3 & 4 & 5 & 6 & 7 & 8 & 9 \\
\hline$\overline{\overline{P_{L}}}$ & 1316,80 & 1246,60 & 1746,50 & 780,12 & 740,75 & 1634,60 & 1289,30 & 1497,30 & 1136,80 \\
\hline$\overline{P_{A}}$ & 3069,00 & 2512,10 & 3406,10 & 2075,00 & 2540,00 & 2227,60 & 1891,30 & 2545,90 & 1450,10 \\
\hline $\bar{A}_{\text {PDI }}[\%]$ & 42,91 & 49,62 & 51,28 & 37,60 & 29,16 & 73,38 & 68,17 & 58,81 & 78,33 \\
\hline Agregado & 10 & 11 & 12 & 13 & 14 & 15 & 16 & 17 & 18 \\
\hline$\overline{P_{L}}$ & 719,62 & 1286,60 & 1640,90 & 266,12 & 683,87 & 1333,33 & 1184,50 & 1004,50 & 1287,30 \\
\hline$\frac{L}{P_{A}}$ & 2021,40 & 1935,50 & 3167,60 & 2296,30 & 1245,00 & 3011,40 & 2013,60 & 2453,10 & 2587,40 \\
\hline $\bar{A}_{P D I}[\%]$ & 35,60 & 66,47 & 51,80 & 11,59 & 54,93 & 44,28 & 58,82 & 40,95 & 49,75 \\
\hline Agregado & 19 & 20 & 21 & 22 & 23 & 24 & 25 & 26 & 27 \\
\hline$\overline{\overline{P_{L}}}$ & 1385,50 & 1016,40 & 1881,00 & 1883,00 & 1451,10 & 996,75 & 852,00 & 1227,40 & 935,12 \\
\hline$\overline{P_{A}}$ & 3330,50 & 2223,40 & 2665,90 & 3163,10 & 2593,40 & 2207,80 & 1705,00 & 1946,90 & 1913,00 \\
\hline $\bar{A}_{P D I}[\%]$ & 41,60 & 45,71 & 70,56 & 59,53 & 55,95 & 45,15 & 49,97 & 63,04 & 48,88 \\
\hline Agregado & 28 & 29 & 30 & 31 & 32 & 33 & 34 & 35 & 36 \\
\hline$\overline{\overline{P_{L}}}$ & 1008,40 & 2056,40 & 657,12 & 889,70 & 706,75 & 526,50 & 335,25 & 1977,70 & 576,62 \\
\hline$\frac{L}{P_{A}}$ & 2015,80 & 2909,60 & 1534,30 & 1451,50 & 1737,10 & 1478,40 & 1141,90 & 3098,30 & 2060,90 \\
\hline $\bar{A}_{P D I}[\%]$ & 50,02 & 70,68 & 42,83 & 61,30 & 40,69 & 35,61 & 29,36 & 63,83 & 27,98 \\
\hline \multicolumn{2}{|c|}{ TOTAL } & \multicolumn{3}{|c|}{$\overline{P_{L}}=41158,22$} & \multicolumn{2}{|c|}{$\overline{P_{A}}=81625,20$} & \multicolumn{3}{|c|}{ Área $=50,42$} \\
\hline
\end{tabular}

Torquato e Silva et al. (2014) utilizaram o método de identificação de outliers como sendo todos os valores fora de 2 desvios padrões $(S)$ da média $(\bar{x})$. Por sua vez, Mahoney (2016) usou o Teste Tau $(\tau)$ de Thompson modificado para identificar outliers em medidas de densidades de núcleos extraídos do revestimento asfáltico após a compactação. Esse teste é recomendado pela Measurement Uncertainty, Part I, PTC 19.1-1998, da American Society of Mechanical Engineers, sempre que houver incertezas, decorrentes de erros aleatórios e de erros sistemáticos.

Escolheu-se o Teste Tau de Thompson Modificado por que ele considera o tamanho da amostra e a cada outlier retirado é feita uma nova iteração com a média e o desvio padrão da nova amostra. 0 Tau de Thompson para cada indivíduo pode ser determinado pela Equação 2. 


$$
\tau_{i}=\frac{\delta_{1}}{S}=\frac{\left|x_{i}-\bar{x}\right|}{S}
$$

Os valores de referência de $\tau$ são calculados a partir da Equação 3, onde $t_{\alpha, n-2}$ é a estatística da distribuição $t$ de Student para um nível de significância $(\alpha)$ e $(n-2)$ graus de liberdade .

$$
\tau_{\alpha, n}=\frac{t_{\alpha, n-2}(n-1)}{\sqrt{n} \cdot \sqrt{n-2+(\alpha, n-2)^{2}}}
$$

Para cada iteração, todos os valores de $\tau_{i}$ da amostra são comparados com um valor de $\tau_{\alpha, n}$. Para a amostra em questão, foram realizadas 2 iterações com $\alpha=0,05 / n=36$ e $\alpha=0,05 / n=35$, sendo o $\tau$ correspondente com valores iguais a $\tau=1,9198$ e $\tau=1,9186$, respectivamente. Todos os valores de $\tau_{i}$ superiores a $\tau_{\alpha, n}$ são considerados outliers. Para essas condições, é possível concluir com 95\% de confiança que o agregado 13 e o agregado 9 têm indícios de serem outliers, visto que seus valores de $\tau_{i}$ foram superiores a $\tau_{\alpha, n}$, conforme Tabela 5 .

Tabela 5: Teste estatístico de Thompson modificado para determinação de outliers

\begin{tabular}{cccccc}
\hline \multicolumn{2}{c}{ Antes da retirada do 10 Outlier } & Após da retirada do 10 Outlier & \multicolumn{2}{c}{ Após da retirada do 2o Outlier } \\
\hline Média $(\bar{x})$ & 0,5042 & Média $(\bar{x})$ & 0,5155 & Média $(\bar{x})$ & 0,5105 \\
Mediana & 0,4986 & Mediana & 0,4997 & Mediana & 0,4904 \\
Desvio Padrão $(S)$ & 0,1455 & Desvio Padrão $(S)$ & 0,1312 & Desvio Padrão $(S)$ & 0,1246 \\
C.V. & 0,2885 & C.V. & 0,2551 & C.V. & 0,2440 \\
\hline$\tau$ (1" iteração) $=1,9198$ & \multicolumn{2}{c}{$\tau_{13}$ (Outlier) $=2,6691$} & $\tau\left(2^{\text {a }}\right.$ iteração $)=1,9186$ & $\tau_{9}$ (Outlier $)=2,0418$ \\
\hline
\end{tabular}

Segundo o teste de verificação de outliers para a amostra analisada, na $1^{\mathrm{a}}$ iteração o agregado 13 foi considerado um outlier, conforme Figura 11 (a). Na 2 $2^{\text {a }}$ iteração o agregado 9 foi considerado outlier, conforme Figura 11 (b).

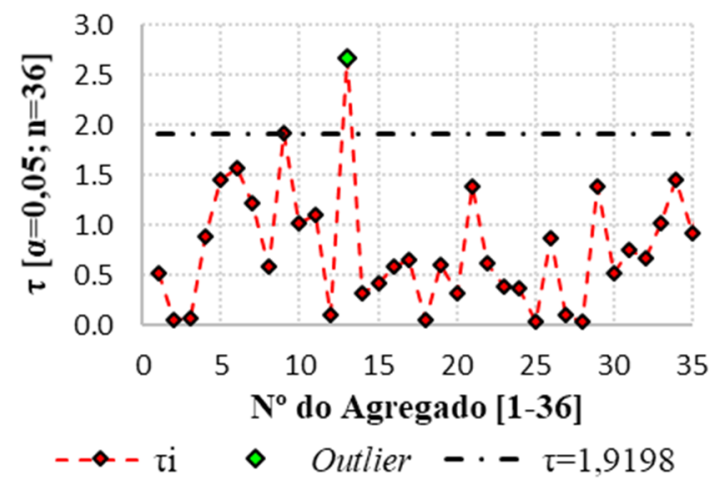

(a)

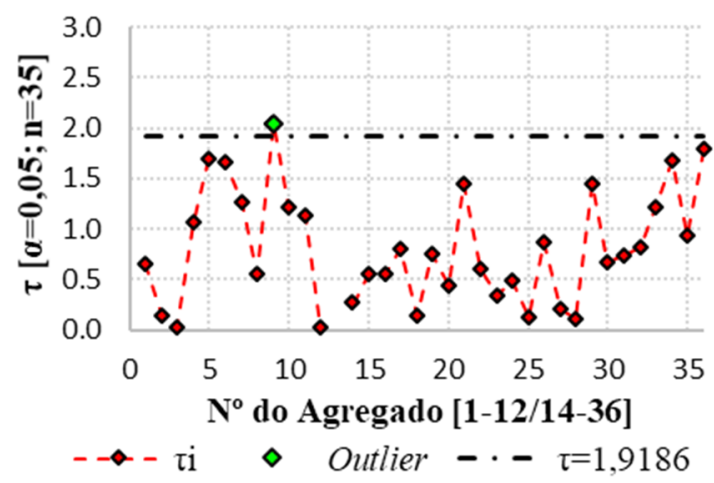

(b)

Figura 11. Análise de outliers.

\section{CONCLUSÕES}

A determinação do percentual de área do agregado recoberto pelo ligante asfáltico, após o ensaio descrito na NBR 12583-2017, se mostrou possível. Os resultados apresentados permitem as seguintes conclusões:

1. Os valores de percentual de área recoberta obtidos por PDI foram muito próximos aos percentuais de área recoberta teóricos das 5 imagens usadas para comparação.

2. O código implementado para contagem de pixels independe da forma da figura geométrica analisada. 
3. A escolha manual dos limiares não se mostrou um forte interferente dos resultados, visto que para um limiar variável de 0,05 os percentuais de área recoberta variaram em média $0,8 \%$.

4. As imagens são geradas em ângulos levemente distintos a cada fotografia e os resultados não se mostraram sensíveis a essa variação.

5. O PDI se mostrou uma ferramenta promissora na análise de misturas asfálticas, em especial na análise da adesividade entre agregados e ligante, podendo quantificar a adesividade de diferentes combinações de agregados e de ligantes asfálticos.

6. Em trabalhos futuros, pretende-se estudar os agregados com recobrimento discrepante (outliers) para investigar se suas propriedades físico-químicas (angularidade, textura, porosidade; teores de sílica e de óxido de cálcio) são distintas dos demais. Espera-se ainda poder relacionar os valores obtidos por PDI com outros ensaios relacionados ao tema, como o Dano por Umidade Induzida e ensaios do tipo pull-off (como o Asphalt Bond Strength).

\section{REFERÊNCIAS}

ABEDA (2010) Aditivos orgânicos melhoradores de adesividade - Usos e benefícios. Informativo Técnico No 1-Junho 2010 Comissão Técnica da Abeda. Associação Brasileira das Empresas Distribuidoras de Asfaltos.

ALBUQUERQUE, M. P.; E. S. CANER; A. G. MELLO e M. P. ALBUQUERQUE (2012) Análise de Imagens e Visão Computacional. Centro Brasileiro de Pesquisas Físicas. Rio de Janeiro.

ARAÚJO, V. M. C.; I. S. BESSA e V. T. F. CASTELO BRANCO (2014) Avaliação da Textura de Misturas Asfálticas a Partir do Uso do Processamento Digital de Imagens. XXVIII Congresso de Pesquisa e Ensino em Transportes - Anpet, Curitiba, PR.

AURA CONCI, E. A. e F. R. LETA (2008) Composição gráfica. Elsevier, $2^{2}$ ed.

BESSA, I. S.; V. T. F. CASTELO BRANCO e J. B. SOARES (2011) Avaliação da Resistência ao Polimento e a Degradação de Agregados Utilizando a Abrasão Los Angeles e o Processamento Digital de Imagens. Anais do XII Congresso Brasileiro de Rodovias e Concessões, ABCR, Foz do Iguaçu.

CAVALCANTE, T. (2010) Técnica para segmentação automática de vias aéreas em imagens de tomografia computadorizada do tórax. Programa de Pós-graduação em Engenharia de Teleinformática. Universidade Federal do Ceará. Fortaleza. Disponível em: http://www.dominiopublico.gov.br/download/texto/cp152023.pdf (acesso em: 10/12/2017).

CHO D. W.; K. KIM (2009) The Mechanisms of Moisture Damage in Asphalt Pavement by Applying Chemistry Aspects. KSCE Journal of Civil Engineering, v. 14, n. 3, p. 333-342. DOI: 10.1007/s12205-010-0333-z.

CURTIS, C. W.; K. ENSLEY e J. EPPS (1993) Fundamental Properties of Asphalt Aggregate Interactions Including Adhesion and Adsorption. SHRP-A-341, Strategic Highway Research Program, National Research Council, Washington, USA.

GONZALES, R. C. e R. E. WOODS (2010) Processamento Digital de Imagens. Always Learning, Pearson, $3^{\mathrm{a}}$ ed.

GOUVEIA, L. T.; F. E. STELLE; L. J. SENGER e E. A. MARTINS FILHO (2015) Esqueletização multiescala para análise de forma de agregados. Revista Transportes, v. 23, p. 14-21. DOI: 10.14295/transportes.v23i2.788.

HAMEDIA, G. H. e S. A. TAHAMIB (2018) The effect of using anti-stripping additives on moisture damage of hot mix asphalt. International Journal of Adhesion and Adhesives, v. 81, p. 90-97. DOI:10.1016/j.ijadhadh.2017.03.016.

HUANG, W. e L. QUAN (2016) Investigation of using binder bond strength test to evaluate adhesion and self-healing properties of modified asphalt binders. Transportation Research Board 95 th Annual Meeting. DOI:10.1016/j.conbuildmat.2016.03.047.

LIANG, R. Y. (2008) “Refine AASHTO T283 resistance of compacted bituminous mixture to moisture induced damage for superpave”. Final Rep. FHWA/OH-2008-1, FHWA, Washington, DC.

LOTTMAN, R. P. (1978) NCHRP Report 192: Predicting Moisture-Induced Damage to Asphaltic Concrete. Transportation Research Board, National Research Council, p. 24. Washington, DC, USA.

LUCAS JÚNIOR, J. L. O. (2018) Influência da adesividade agregado-ligante no trincamento por fadiga de misturas asfálticas. Dissertação de Mestrado, Programa de Pós-Graduação em Engenharia de Transportes, Universidade Federal do Ceará, Fortaleza. Disponível em: http://www.repositorio.ufc.br/handle/riufc/34068 (acesso em: 05/12/2018).

MAHONEY, S. Z. J. (2016) Reviewing the Testing Protocol for Density Cores Collected from CTDOT during the 2012 and 2013 Construction Seasons. Research Project: SPR-2281, Connecticut Department of Transportation Bureau of Policy and Planning Roadway Information Systems Unit Research Section.

MARQUES FILHO, O. e H. VIEIRA NETO (1999) Processamento digital de imagens. Brasport, Rio de Janeiro.

NBR 12583 (2017) Agregado Graúdo - Determinação da adesividade ao ligante betuminoso. Associação Brasileira de Normas Técnicas.

OTSU, N. (1979) A Threshold Selection Method from Gray-Level Histograms. IEEE Transactions on Systems, Man, and Cybernetics, v. 9, n. 1, p. 62-66. 
PERES, L. M. (2010) Aplicação de Processamento Digital de Imagens a Problemas de Engenharia Civil. Graduação em engenharia Civil. Faculdade de Engenharia da UFJF, Juiz de Fora. Disponível em: http://www.ufjf.br/mac/files/2009/05/TFCLeandroMota.pdf (acesso em 10/12/2017)

SILVA, A. M. Curso Processamento digital de imagens de satélite. Centro de Eventos da PUCRS, 2001. Disponível em: < http://www.cartografia.org.br >. Acesso em: 19/09/2017.

SONKA, M; V. HLAVAC e R. BOYLE (1998) Image Processing, analysis, and Machine Vision. Brooks/Cole Publishing Company. USA.

TORQUATO E SILVA, S. A.; J. B. SOARES; A. S. HOLANDA e J. A. OLIVEIRA (2014) Utilização do Método dos Elementos Finitos em Retroanálise de Pavimentos Flexíveis. In: XXVIII Congresso de Pesquisa e Ensino em Transportes - Anpet, Curitiba, PR.

VASCONCELOS, K. L.; F. EVANGELISTA JR. e J. B. SOARES (2005) Análise da Estrutura Interna de Misturas Asfálticas. In: XVII Congresso Brasileiro de Pesquisa e Ensino em Transportes, Recife, PE.

ZAITOUNA, N. M. e M. J. AQELB (2015) Survey on Image Segmentation Techniques. International Conference on Communication. Management and Information Technology. DOI: 10.1016/j.procs.2015.09.027.

ZELELEW, H. M. e A. T. PAPAGIANNAKIS (2011) A volumetrics thresholding algorithm for processing asphalt concrete X-ray CT images. International Journal of Pavement Engineering, v. 12, n. 6, p. 543-551. DOI: 10.1080/10298436.2011.561345.

ANEXO 


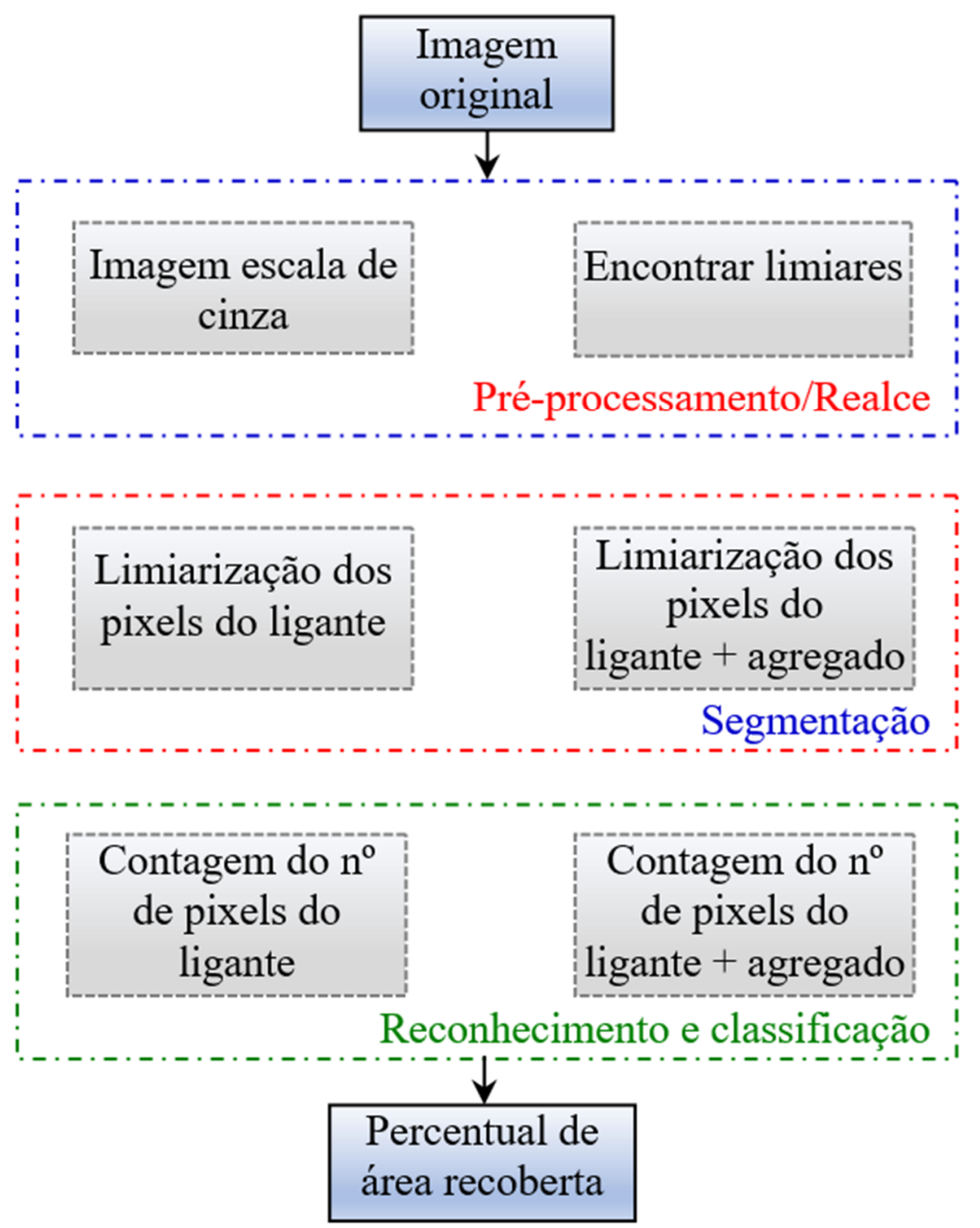

Figura A1. Fluxograma da metodologia de PDI. 
I=imread('TESTE.jpg');

figure(1); imshow(I);

$\mathrm{EC}=\operatorname{rgb} 2 \operatorname{gray}(\mathrm{I})$;

figure (2); imshow(EC);

figure (3); imhist(EC);

level $=0.200$;

$\mathrm{A}=\mathrm{im} 2 \mathrm{bw}(\mathrm{EC}$, level) ;

figure(4); imshow(A);

$\mathrm{B}=\sim \mathrm{A}$;

figure(5); imshow(B);

bwarea(B);

$\mathrm{P}_{\mathrm{L}}=\mathrm{bwarea}(\mathrm{B})$

$\mathrm{H}=$ imread('TESTE.jpg');

figure(6); imshow(H);

$\mathrm{EC} 1=\operatorname{rgb} 2 \operatorname{gray}(\mathrm{H})$;

imshow(EC1);

figure (7); imhist(EC1);

level $=0.785$;

$\mathrm{C}=\mathrm{im} 2 \mathrm{bw}(\mathrm{EC} 1$, level $)$

figure(8); imshow(C);

$\mathrm{D}=\sim \mathrm{C}$;

figure(9); imshow(D);

bwarea(D);

$\mathrm{P}_{\mathrm{A}}=\mathrm{bwarea}(\mathrm{D})$

$\mathrm{A}_{\mathrm{PDI}}=\mathrm{P}_{\mathrm{L}} / \mathrm{P}_{\mathrm{A}}$

Figura A2. Código da programação. 